\title{
Explaining the Flavour Anomalies with the Pati-Salam Vector Leptoquark*
}

\author{
Andreas Crivellin ${ }^{\dagger+}$ \\ Paul Scherrer Institut, CH-5232 Villigen PSI, Switzerland \\ E-mail: andreas.crivellinepsi.ch
}

\begin{abstract}
In recent years several experiments revealed intriguing hints for new physics (NP) in $B$ meson decays involving $b \rightarrow c \tau \nu$ and $b \rightarrow s \ell^{+} \ell^{-}$transitions at the $4-5 \sigma$ level. These signs for NP are supported by slight disagreements in the analogous observables with $b \rightarrow u \tau \nu$ and $b \rightarrow d \mu^{+} \mu^{-}$ transitions. While not significant on their own, these processes point in the same direction.

Since all these observables are semi-leptonic, leptoquarks are prime candidates for a solution to these puzzels since they give tree-level effects here while their contributions to other flavour observables, which agree with the SM predictions, are loop suppressed.

In these proceedings we focus on the vector leptoquark $S U(2)$ singlet which provides a natural explanations for both the $b \rightarrow c(u) \tau \nu$ and the $b \rightarrow s(d) \mu^{+} \mu^{-}$data. However, to account for the preferred NP effect in $b \rightarrow c(u) \tau \nu$ large couplings to $\tau$ leptons are necessary and loop effects become important. Including them in the phenomenological analysis, the fit to data is even improved.

Finally, we review two UV complete models which give rise to the desired vector leptoquark $S U(2)$ singlet. The first possibility is to add either three generations of vector-like fermions to the original Pati-Salam model and break the symmetry with a Higgs mechanism. Alternatively, it is possible to break the PS gauge group to the SM one on a compact extra dimension like in Randall Sundrum models.
\end{abstract}

Sixth Annual Conference on Large Hadron Collider Physics (LHCP2018)

4-9 June 2018

Bologna, Italy

\footnotetext{
* Matches my previous PoS proceedings for ALPS2018.

${ }^{\dagger}$ Speaker.

$¥$ I thank the organizers for the invitation and the opportunity to present my research here. This work is supported by an Ambizione Grant of the Swiss National Science Foundation (PZ00P2_154834) and an explora grant (FPA201461478-EXP).
} 


\section{Introduction}

Even though the LHC did not directly observe any particles beyond the ones of Standard Model (SM), intriguing hints for lepton flavour universality (LFU) violating NP in semi-leptonic $B$ decays were acquired:

$$
b \rightarrow s(d) \ell^{+} \ell^{-}
$$

In these flavour changing neutral current processes (which are only generated at the loop level within the SM) the ratios $R\left(K^{(*)}\right)=\frac{B \rightarrow K^{(*)} \mu^{+} \mu^{-}}{B \rightarrow K^{(*)} e^{+} e^{-}}$[1]( [2]) indicate LFU violation with a combined significance of $\approx 4 \sigma$ [3-8]. Taking also into account all other $b \rightarrow s \mu^{+} \mu^{-}$observables (like $P_{5}^{\prime}$ [9]), the global fit even shows compelling evidence for NP $(>5 \sigma)$ [10]. Concerning $b \rightarrow$ $d \ell^{+} \ell^{-}$transitions, the theoretical analysis of Ref. [11] shows that the LHCb measurement of $B \rightarrow$ $\pi \mu^{+} \mu^{-}$[12] slightly differs from the theory expectation. Even though not being significant on its own, the central value is agrees with the expectation from $b \rightarrow s \ell^{+} \ell^{-}$since the effect is of same order and has the same sign as in $b \rightarrow s \ell^{+} \ell^{-}$, compared to the SM.

$$
b \rightarrow c(u) \tau v
$$

For these charged current processes (which are mediated in the SM by a tree-level $W$ exchange) the ratios $R\left(D^{(*)}\right)=\frac{B \rightarrow D^{(*)} \tau v}{B \rightarrow D^{(*)} \ell v}$ (with $\ell=e, \mu$ ) measure again LFU violation. They differ in combination from their SM predictions by $\approx 4 \sigma$ [13]. Also, the ratio $R(J / \Psi)=\frac{B_{c} \rightarrow J / \Psi \tau v}{B_{c} \rightarrow J / \Psi \mu v}$ [14] exceeds the SM prediction in agreement with the expectations from $R\left(D^{(*)}\right)[15,16]$. Concerning the analogous $b \rightarrow$ $u \tau v$ transitions, the SM prediction for $B \rightarrow \tau v$ depends on $V_{u b}$ where previous lattice calculations resulted in quite small values of $V_{u b}$ while recent calculations give larger ones (see Ref. [17] for an overview). However, the measurement is still above the SM prediction as can be seen from the global fit [18]. In the ratio $R(\pi)=\frac{B \rightarrow \pi \tau v}{B \rightarrow \pi \ell v}$ there is a slight disagreement between the theory prediction [19] and the experimental measurement [20] as well, which however does not depend on $V_{u b}$. Again, these results are not significant on their own, but are within the expectation from $b \rightarrow c \tau v$ assuming that NP scales like the SM as $V_{u b} / V_{c b}$.

The vector leptoquark (LQ) $S U(2)_{L}$ singlet with hypercharge $-4 / 3$ arising in the famous PatiSalam model [21] is capable of explaining all these anomalies [22-28] and therefore several attempts to construct a UV completion for this LQ to address the anomalies have been made [29-41]. It can give a sizable effect in $b \rightarrow c(u) \tau \nu$ data without violating bounds from $b \rightarrow s(d) v \bar{v}$ and/or direct searches, provides (at tree level) a $C_{9}=-C_{10}$ solution to $b \rightarrow s \ell^{+} \ell^{-}$data and does not lead to proton decay at any order in perturbation theory.

\section{Simplified Model for the Vector Leptoquark}

Let us start the phenomenological study by considering a simplified model. It is obtained by extending the SM by a vector LQ $S U(2)_{L}$ singlet with hypercharge $-4 / 3$, mass $M$ and interactions with fermions determined (before EW symmetry breaking) by

$$
\mathscr{L}_{V^{\mu}}=\left(\kappa_{f i}^{L} \overline{Q_{f}} \gamma_{\mu} L_{i}+\kappa_{f i}^{R} \overline{d_{f}} \gamma_{\mu} e_{i}\right) V_{1}^{\mu \dagger}+\text { h.c. } .
$$

After EW symmetry breaking, we work in the down basis, i.e. no CKM elements appear in flavour changing neutral currents of down quarks. With these conventions at hand, we now study the phenomenology taking into account the loop effects calculated in Ref. [43]. 

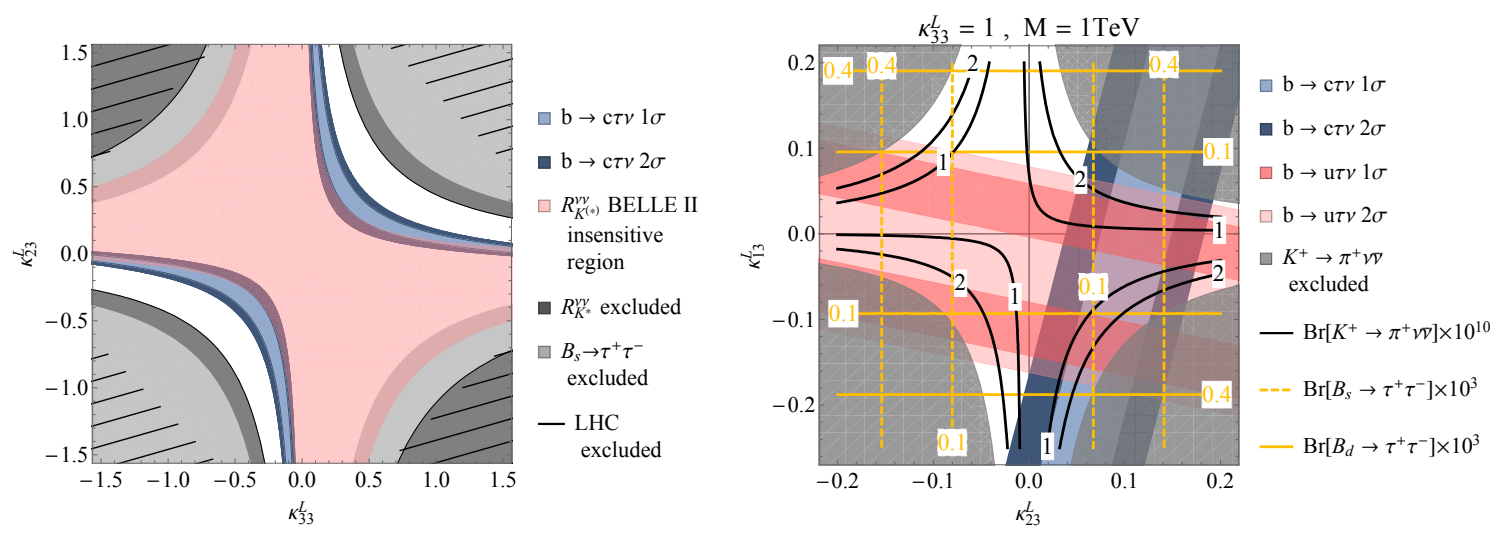

Figure 1: Left: Regions in the $\kappa_{23}^{L}-\kappa_{33}^{L}$ plane for $M=1 \mathrm{TeV}$ : The blue regions are preferred by $b \rightarrow c \tau \nu$ data, the (light) grey region is excluded by $\left(B_{s} \rightarrow \tau^{+} \tau^{-}\right) B \rightarrow K^{*} v v$ and the hatched region indicates the (approximate) excluded region by LHC, obtained by a naive rescaling of the PDFs using the bounds of Ref. [44]. Within the light red region $\operatorname{Br}\left[B \rightarrow K^{*} v v\right]$ is smaller than the expected BELLE II sensitivity. Right: Predictions for $B_{q} \rightarrow \tau^{+} \tau^{-}$and $K \rightarrow \pi v \bar{v}$ (contour lines) in the $\kappa_{13}^{L} \kappa_{23}^{L}$ plane for $M=1 \mathrm{TeV}$ and $\kappa_{33}^{L}=1$. The colored regions are preferred by $b \rightarrow c(u) \tau \nu$ data (where we naively averaged $R\left(D^{(*)}\right)$ and $R(J / \Psi)$ or $R(\pi)$ and $B \rightarrow \tau \nu$, respectively). The gray region is excluded by $K^{+} \rightarrow \pi^{+} \nu \bar{v}$. Here we assumed all couplings $\kappa_{i j}^{L}$ to be real.

Assuming that $\kappa_{23}^{L}$ and $\kappa_{33}^{L}$, which are necessary to explain $b \rightarrow c \tau \nu$ data, are the only non-zero couplings, we show in Fig. 1 that $b \rightarrow c \tau v$ data can be explained for a LQ mass of $1 \mathrm{TeV}$ without violating bounds from $B_{s} \rightarrow \tau^{+} \tau^{-}, B \rightarrow K^{(*)} v \bar{v}$ or direct LHC searches.

Taking into account the effect of $\kappa_{13}^{L}$ in addition, many correlations arise. First of all, $b \rightarrow$ $c(u) \tau \nu$ is already at tree level correlated to $b \rightarrow s(d) \tau^{+} \tau^{-}$. In addition, the $W$ boxes generate effects in $B \rightarrow K^{(*)}(\pi) \nu \bar{v}$ and $K \rightarrow \pi \nu \bar{v}$. While the bounds from $B \rightarrow K^{(*)}(\pi) \nu \bar{v}$ turn out to be weaker than the ones from $B_{q} \rightarrow \tau^{+} \tau^{-}$, there are striking correlations with $K \rightarrow \pi \nu \bar{v}$ as can be seen from the right plot in Fig. 1.

Now we allow in addition non-vanishing couplings $\kappa_{32}^{L}$ and $\kappa_{22}^{L}$, obtaining tree level effects in $b \rightarrow s \mu^{+} \mu^{-}$. Interestingly, due to the loop effects originating from the $b \rightarrow c \tau \nu$ explanation, one predicts a flavor universal effect in $C_{9, s b}^{\ell \ell}$ and $C_{7}^{s b}$ which is supplemented by the the tree-level effect in $C_{9, s b}^{\mu \mu}=-C_{10, s b}^{\mu \mu}$ with muons only. This means that the relative NP effect compared to the SM in $P_{5}^{\prime}$ should be larger than in $R\left(K^{(*)}\right)$ which is in perfect agreement with the global fit.

\section{Possible UV completions}

\subsection{Generations of vector-like fermions}

Our starting point is the Pati-Salam model [21] with the gauge group $S U(4) \times S U(2)_{L} \times$ $S U(2)_{R}$. Thus, left-handed fermions transform as $S U(2)_{L}$ doublets while right-handed ones form $S U(2)_{R}$ doublets. This necessarily leads to the introduction of three right-handed neutrinos. In our model [33], we extend the fermion content of the original model having now 6 fermion fields $X_{i}^{L, R}, Y_{i}^{L, R}, Z_{i}^{L, R}$ which are all in the fundamental representation of $S U(4)$ as well as (at least) two 
more Higgs fields $\Sigma^{1,2}$ (see Ref. [33] for details). One does not need to explicitly specify the EW symmetry breaking sector since we know that due to the decoupling theorem, the symmetry breaking sector must reduce, in the limit of heavy additional Higgses, to one light $S U(2)$ doublet with vev $v$ giving rise the chiral fermion and weak gauge boson masses. This approach is model independent in the sense that including additional physical Higgses would imply focussing on a specific UV realization of the model. The predictions are very much the same as in the model independent approach studied above. Furthermore, the bound from $B_{s}-\bar{B}_{s}$ mixing (which cannot be reliably calculated in the simplified model) is respected due to a GIM-like mechanism of the lepton and their vector-like partners.

\subsection{Randall Sundrum}

Here we review the model of Ref. [36]. Within the 5D RS space-time [45] the PS symmetry group is a bulk gauge symmetry. The symmetry is broken to its SM subgroup by boundary conditions imposed on the UV brane. As in the standard PS model, all fermions are embedded into complete representations of the PS gauge group. In addition, they form bulk fields in the RS background with the zero modes corresponding to the SM quarks and leptons. Their localizations are in principle determined by their 5D bulk masses [46] but can be altered by the presence of brane-kinetic terms. Since on the UV brane only the SM gauge symmetry is unbroken, the localizations of quarks and leptons can differ from each other. The Higgs doublet forms a 4D field confined to the UV brane with couplings to the KK modes which are strongly suppressed, ensuring the compatibility with electroweak precision tests.

The dual theory in 4 dimensions, according to the AdS/CFT theorem [47], is a composite model with a global PS symmetry and KK modes corresponding to composite resonances. The gauging of the SM group explicitly breaks the global symmetry within the elementary sector. Therefore, the SM fermions are partially composite with a linear mixing of the elementary fermions with composite ones of the same quantum numbers. Thus, the deconstructed version of our model [48] contains composite vector resonances of the $S U(4) \times S U(2)_{L} \times S U(2)_{R}$ symmetry group with common mass $M$ and three generations of heavy vector-like quarks and leptons corresponding to the first KK modes. We denote the mixing angles between composite and fundamental fermions $f=u, d, \ell$ of flavour $i s_{i}^{f}=\sin \alpha_{i}^{f}$ and $s_{\ell}$ parametrizes the misalignment in flaovur space between the leptons of the second and third generation.

Again, concerning the phenomenology, we want large effect in $b \rightarrow c \tau v$ and thus a sizable compositeness of the third generation is necessary. The scale of compositeness $M$ should not be too high and we choose $3 \mathrm{TeV}$. In order to get the necessary effect in $b \rightarrow s \mu^{+} \mu^{-}$, while not violating the upper limit on the $\tau \rightarrow 3 \mu$ branching ratio, moderate values of $s_{2}^{\ell}$ (the degree of compositeness of the second generation) are preferred. In the left plot of Fig. 2 we show the allowed regions in the $s_{2}^{q}-s_{\ell}$ plane for $s_{2}^{\ell}=0.2, s_{3}^{\ell}=1 / \sqrt{2}$ and $s_{3}^{q}=\sqrt{3} / 2$ where with $R(X) / R(X)_{\mathrm{SM}} \approx 1.07$. Due to the small coupling to muons (compared to the one to taus leptons) NP effects in $b \rightarrow c \mu v$ are found to be below the permille level and are therefore consistent with current data [49]. One can see that $b \rightarrow s \mu^{+} \mu^{-}$can be explained at the $1 \sigma$ level without violating the bounds from $D-\bar{D}$ mixing or $\tau \rightarrow 3 \mu$. In the right plot of Fig. 2 we depict the correlations between $R(X) / R(X)_{\mathrm{SM}}$ and $b \rightarrow s \mu^{+} \mu^{-}$by scanning over $s_{3}^{q}, s_{2}^{q}, s_{3}^{\ell}, s_{2}^{\ell}$ and $s_{\ell}$. Only the points in parameter space which are consistent with all experimental bounds are shown. One can see that a large effect in $b \rightarrow s \mu^{+} \mu^{-}$ 

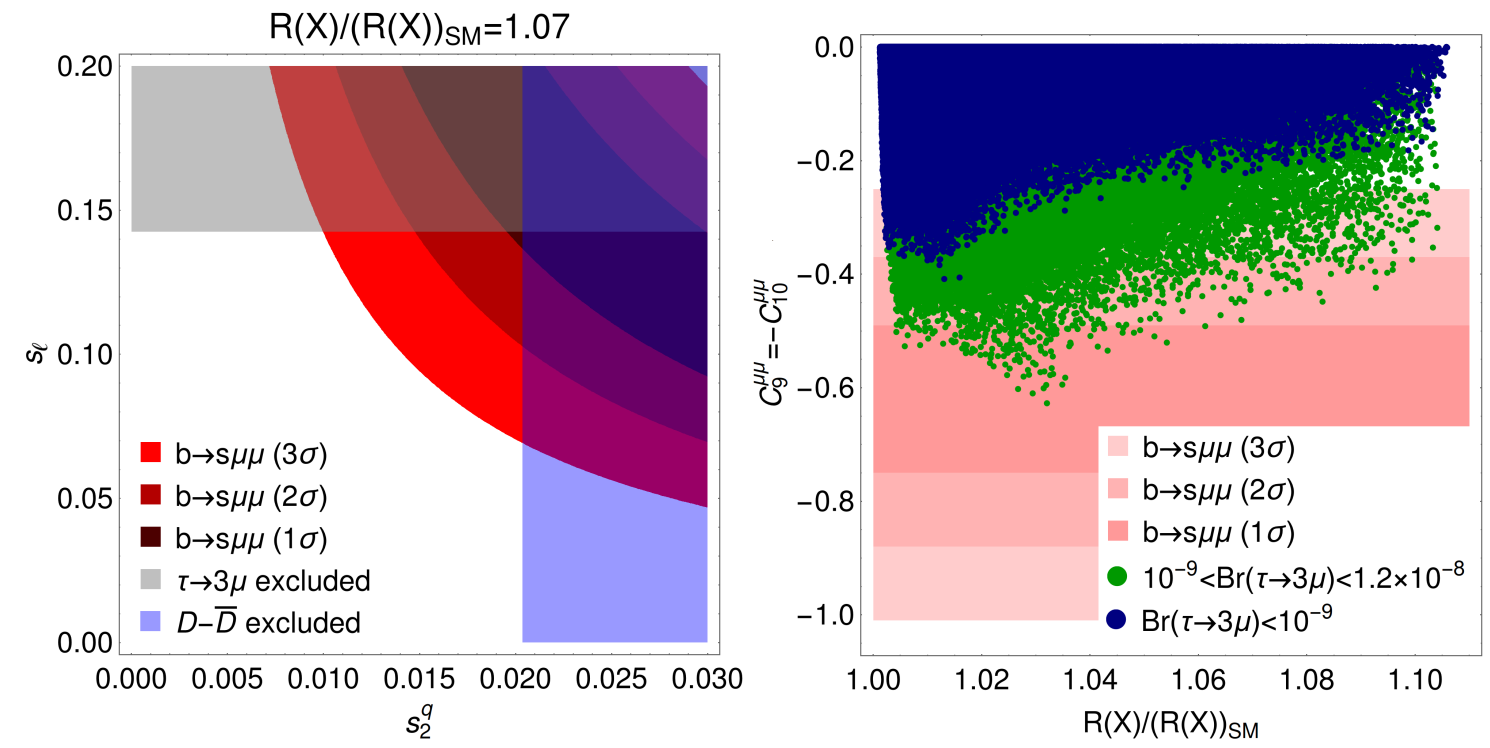

Figure 2: Left: Allowed regions from $b \rightarrow s \mu^{+} \mu^{-}$(red) and the exclusion limits from $D-\bar{D}$ mixing (blue) and $\tau \rightarrow 3 \mu$ (gray) for $M=3 \mathrm{TeV}, s_{2}^{\ell}=0.2, s_{3}^{\ell}=1 / \sqrt{2}$ and $s_{3}^{q}=\sqrt{3} / 2$. With these values, $R(X) / R(X)_{\mathrm{SM}} \approx$ 1.07 (with $X=D, D^{*}, J / \Psi$ ). We see that $b \rightarrow s \mu^{+} \mu^{-}$can be explained at the $1 \sigma$ level without violating the bounds from other observables. Right: Correlations between $R(X) / R(X)_{\mathrm{SM}}$ and $b \rightarrow s \mu^{+} \mu^{-}$for $M=3 \mathrm{TeV}$. Here we scanned over $0.3<s_{3}^{q}<\sqrt{3} / 2,0<s_{2}^{q}<0.2,0.3<s_{3}^{\ell}<\sqrt{3} / 2,0<s_{2}^{\ell}<0.2$ and $0<s_{\ell}<0.3$. Only the parameter points consistent with $D-\bar{D}$ mixing are shown. As we can see, the predicted branching ratio for $\tau \rightarrow 3 \mu$ is large and very well within the reach of Belle II.

limits the size of the possible effect in $R(X) / R(X)_{\mathrm{SM}}$ and vice versa. Furthermore, the solution of the $b \rightarrow s \mu^{+} \mu^{-}$anomaly predicts a large branching ratio for $\tau \rightarrow 3 \mu$ which is within the reach of Belle II. Due to the constraints from $D^{0}-\bar{D}^{0}$ mixing and $\tau \rightarrow 3 \mu$ one does not obtain sizeable effects in $b \rightarrow s \tau \mu$. However, the constraint from $D^{0}-\bar{D}^{0}$ mixing could be relaxed by making the first and second generation equally composite [32].

\section{References}

[1] R. Aaij et al. [LHCb Collaboration], Phys. Rev. Lett. 113 (2014) 151601 doi:10.1103/PhysRevLett.113.151601 [arXiv:1406.6482 [hep-ex]].

[2] R. Aaij et al. [LHCb Collaboration], JHEP 1708 (2017) 055 doi:10.1007/JHEP08(2017)055 [arXiv:1705.05802 [hep-ex]].

[3] W. Altmannshofer, P. Stangl and D. M. Straub, Phys. Rev. D 96 (2017) no.5, 055008 doi:10.1103/PhysRevD.96.055008 [arXiv:1704.05435 [hep-ph]].

[4] G. D'Amico, M. Nardecchia, P. Panci, F. Sannino, A. Strumia, R. Torre and A. Urbano, JHEP 1709 (2017) 010 doi:10.1007/JHEP09(2017)010 [arXiv:1704.05438 [hep-ph]].

[5] M. Ciuchini, A. M. Coutinho, M. Fedele, E. Franco, A. Paul, L. Silvestrini and M. Valli, Eur. Phys. J. C 77 (2017) no.10, 688 doi:10.1140/epjc/s10052-017-5270-2 [arXiv:1704.05447 [hep-ph]].

[6] G. Hiller and I. Nisandzic, Phys. Rev. D 96 (2017) no.3, 035003 doi:10.1103/PhysRevD.96.035003 [arXiv: 1704.05444 [hep-ph]]. 
[7] L. S. Geng, B. Grinstein, S. Jäger, J. Martin Camalich, X. L. Ren and R. X. Shi, Phys. Rev. D 96 (2017) no.9, 093006 doi:10.1103/PhysRevD.96.093006 [arXiv:1704.05446 [hep-ph]].

[8] T. Hurth, F. Mahmoudi, D. Martinez Santos and S. Neshatpour, Phys. Rev. D 96 (2017) no.9, 095034 doi:10.1103/PhysRevD.96.095034 [arXiv:1705.06274 [hep-ph]].

[9] R. Aaij et al. [LHCb Collaboration], JHEP 1602 (2016) 104 doi:10.1007/JHEP02(2016)104 [arXiv:1512.04442 [hep-ex]].

[10] B. Capdevila, A. Crivellin, S. Descotes-Genon, J. Matias and J. Virto, JHEP 1801 (2018) 093 doi:10.1007/JHEP01(2018)093 [arXiv:1704.05340 [hep-ph]].

[11] C. Hambrock, A. Khodjamirian and A. Rusov, Phys. Rev. D 92 (2015) no.7, 074020 doi:10.1103/PhysRevD.92.074020 [arXiv:1506.07760 [hep-ph]].

[12] R. Aaij et al. [LHCb Collaboration], JHEP 1510 (2015) 034 doi:10.1007/JHEP10(2015)034 [arXiv:1509.00414 [hep-ex]].

[13] Y. Amhis et al. [HFLAV Collaboration], Eur. Phys. J. C 77 (2017) no.12, 895 doi:10.1140/epjc/s10052-017-5058-4 [arXiv:1612.07233 [hep-ex]].

[14] R. Aaij et al. [LHCb Collaboration], Phys. Rev. Lett. 120 (2018) no.12, 121801 doi:10.1103/PhysRevLett.120.121801 [arXiv:1711.05623 [hep-ex]].

[15] R. Watanabe, Phys. Lett. B 776 (2018) 5 doi:10.1016/j.physletb.2017.11.016 [arXiv:1709.08644 [hep-ph]].

[16] B. Chauhan and B. Kindra, arXiv:1709.09989 [hep-ph].

[17] G. Ricciardi, Mod. Phys. Lett. A 32 (2017) no.05, 1730005 doi:10.1142/S0217732317300051 [arXiv:1610.04387 [hep-ph]].

[18] J. Charles et al. [CKMfitter Group], Eur. Phys. J. C 41 (2005) no.1, 1 doi:10.1140/epjc/s2005-02169-1 [hep-ph/0406184].

[19] F. U. Bernlochner, Phys. Rev. D 92 (2015) no.11, 115019 doi:10.1103/PhysRevD.92.115019 [arXiv:1509.06938 [hep-ph]].

[20] P. Hamer et al. [Belle Collaboration], Phys. Rev. D 93 (2016) no.3, 032007 doi:10.1103/PhysRevD.93.032007 [arXiv:1509.06521 [hep-ex]].

[21] J. C. Pati and A. Salam, Phys. Rev. D 10 (1974) 275 Erratum: [Phys. Rev. D 11 (1975) 703]. doi:10.1103/PhysRevD.10.275, 10.1103/PhysRevD.11.703.2

[22] R. Alonso, B. Grinstein and J. Martin Camalich, JHEP 1510 (2015) 184 doi:10.1007/JHEP10(2015)184 [arXiv:1505.05164 [hep-ph]].

[23] L. Calibbi, A. Crivellin and T. Ota, Phys. Rev. Lett. 115 (2015) 181801 doi:10.1103/PhysRevLett.115.181801 [arXiv:1506.02661 [hep-ph]].

[24] S. Fajfer and N. Kosnik, Phys. Lett. B 755 (2016) 270 doi:10.1016/j.physletb.2016.02.018 [arXiv:1511.06024 [hep-ph]].

[25] G. Hiller, D. Loose and K. Schönwald, JHEP 1612 (2016) 027 doi:10.1007/JHEP12(2016)027 [arXiv:1609.08895 [hep-ph]].

[26] B. Bhattacharya, A. Datta, J. P. Guevin, D. London and R. Watanabe, JHEP 1701 (2017) 015 doi:10.1007/JHEP01(2017)015 [arXiv:1609.09078 [hep-ph]]. 
[27] D. Buttazzo, A. Greljo, G. Isidori and D. Marzocca, JHEP 1711 (2017) 044 doi:10.1007/JHEP11(2017)044 [arXiv:1706.07808 [hep-ph]].

[28] J. Kumar, D. London and R. Watanabe, arXiv:1806.07403 [hep-ph].

[29] R. Barbieri, G. Isidori, A. Pattori and F. Senia, Eur. Phys. J. C 76 (2016) no.2, 67 doi:10.1140/epjc/s10052-016-3905-3 [arXiv:1512.01560 [hep-ph]].

[30] R. Barbieri, C. W. Murphy and F. Senia, Eur. Phys. J. C 77 (2017) no.1, 8 doi:10.1140/epjc/s10052-016-4578-7 [arXiv:1611.04930 [hep-ph]].

[31] N. Assad, B. Fornal and B. Grinstein, Phys. Lett. B 777 (2018) 324 doi:10.1016/j.physletb.2017.12.042 [arXiv:1708.06350 [hep-ph]].

[32] L. Di Luzio, A. Greljo and M. Nardecchia, Phys. Rev. D 96 (2017) no.11, 115011 doi:10.1103/PhysRevD.96.115011 [arXiv:1708.08450 [hep-ph]].

[33] L. Calibbi, A. Crivellin and T. Li, arXiv:1709.00692 [hep-ph].

[34] M. Bordone, C. Cornella, J. Fuentes-Martin and G. Isidori, Phys. Lett. B 779 (2018) 317 doi:10.1016/j.physletb.2018.02.011 [arXiv:1712.01368 [hep-ph]].

[35] R. Barbieri and A. Tesi, Eur. Phys. J. C 78 (2018) no.3, 193 doi:10.1140/epjc/s 10052-018-5680-9 [arXiv:1712.06844 [hep-ph]].

[36] M. Blanke and A. Crivellin, Phys. Rev. Lett. 121 (2018) no.1, 011801 doi:10.1103/PhysRevLett.121.011801 [arXiv:1801.07256 [hep-ph]].

[37] A. Greljo and B. A. Stefanek, Phys. Lett. B 782 (2018) 131 doi:10.1016/j.physletb.2018.05.033 [arXiv:1802.04274 [hep-ph]].

[38] M. Bordone, C. Cornella, J. Fuentes-MartÃ nn and G. Isidori, arXiv:1805.09328 [hep-ph].

[39] D. Marzocca, JHEP 1807 (2018) 121 doi:10.1007/JHEP07(2018)121 [arXiv:1803.10972 [hep-ph]].

[40] S. Matsuzaki, K. Nishiwaki and K. Yamamoto, arXiv:1806.02312 [hep-ph].

[41] L. Di Luzio, J. Fuentes-Martin, A. Greljo, M. Nardecchia and S. Renner, arXiv:1808.00942 [hep-ph].

[42] B. Capdevila, A. Crivellin, S. Descotes-Genon, L. Hofer and J. Matias, Phys. Rev. Lett. 120 (2018) no.18, 181802 doi:10.1103/PhysRevLett.120.181802 [arXiv:1712.01919 [hep-ph]].

[43] A. Crivellin, M. Ghezzi, L. Panizzi, G. M. Pruna and A. Signer, arXiv:1807.10224 [hep-ph].

[44] D. A. Faroughy, A. Greljo and J. F. Kamenik, Phys. Lett. B 764 (2017) 126 doi:10.1016/j.physletb.2016.11.011 [arXiv:1609.07138 [hep-ph]].

[45] L. Randall and R. Sundrum, Phys. Rev. Lett. 83 (1999) 3370 doi:10.1103/PhysRevLett.83.3370 [hep-ph/9905221].

[46] Y. Grossman and M. Neubert, Phys. Lett. B 474 (2000) 361 doi:10.1016/S0370-2693(00)00054-X [hep-ph/9912408].

[47] J. M. Maldacena, Int. J. Theor. Phys. 38 (1999) 1113 [Adv. Theor. Math. Phys. 2 (1998) 231] doi:10.1023/A:1026654312961, 10.4310/ATMP.1998.v2.n2.a1 [hep-th/9711200].

[48] R. Contino, T. Kramer, M. Son and R. Sundrum, JHEP 0705 (2007) 074 doi:10.1088/1126-6708/2007/05/074 [hep-ph/0612180].

[49] M. Jung and D. M. Straub, arXiv:1801.01112 [hep-ph]. 